\section{OP0094 EULAR-ACR 2019 CLASSIFICATION CRITERIA FOR SLE: CAN WE CLASSIFY USING LABORATORY TESTS ALONE?}

S. Dyball ${ }^{1}$, J. Reynolds ${ }^{2}$, E. Mccarthy ${ }^{3}$, S. Haque ${ }^{4}$, A. Herrick ${ }^{1,5}$, H. Chinoy ${ }^{1,5}$ E. Bruce ${ }^{3}$, B. Parker ${ }^{1,3}$, I. N. Bruce ${ }^{1,3} .{ }^{1}$ The University of Manchester, Manchester, United Kingdom; ${ }^{2}$ University of Birmingham, Birmingham, United Kingdom; ${ }^{3}$ The Kellgren Centre of Rheumatology, Manchester, United Kingdom; ${ }^{4}$ University Hospital of South Manchester, Wythenshawe, United Kingdom; ${ }^{5}$ Salford Royal NHS Foundation Trust, Manchester, United Kingdom

Background: The EULAR-ACR 2019 (EULAR19) classification criteria for systemic lupus erythematosus (SLE) were developed to improve the sensitivity and specificity of previous criteria. Notably, both the EULAR19 and existing SLICCSLE 2012 (SLICC12) criteria can classify patients as having SLE by the presence of immunology and haematological abnormalities in the absence of any signs or symptoms.

Objectives: To validate the EULAR19 criteria, with comparison to existing criteria, in a large cohort of patients with an established systemic autoimmune rheumatic disease (SARD).

Methods: We recruited 227 adult patients who were ANA positive with $\geq 1$ clinical feature suggestive of a SARD, from three hospitals in the North West of England. Clinician diagnosis was used as gold standard; we then applied the EULAR19, SLICC12 and the ACR-SLE 1997 (ACR97) criteria.

Results: Of the 227 patients recruited, by clinician diagnosis, 89 patients (36\%) had SLE, 43 (17\%) primary Sjögren's (pSS), 62 (25\%) undifferentiated CTD (UCTD), 25 (10\%) systemic sclerosis (SSc) and $8(3 \%)$ an inflammatory myositis. The characteristics of these patients and the breakdown of the EULAR19 criteria are outlined in figure 1 .

\begin{tabular}{|l|l|l|l|l|l|}
\hline & SLE n=89 & pSS n=43 & UCTD n=62 & SSc n=25 & Myositis n=8 \\
\hline Female N (\%) & $82(92)$ & $42(98)$ & $53(85)$ & $24(96)$ & $8(100)$ \\
\hline Age, mean (SD) /years & $44(13)$ & $52(12)$ & $47(13)$ & $60(9)$ & $53(7)$ \\
\hline Disease duration, mean (SD) /years & $12(10)$ & $6(5)$ & $5(7)$ & $9(11)$ & $3(3)$ \\
\hline SLE classification criteria & $75(84)$ & $10(23)$ & $20(32)$ & $1(4)$ & 0 \\
\hline EULAR SLE 2019, N (\%) & $77(87)$ & $9(21)$ & $21(34)$ & $2(8)$ & $1(13)$ \\
\hline ACR SLE 1997, N (\%) & $84(94)$ & $15(35)$ & $33(53)$ & $4(16)$ & $2(25)$ \\
\hline SLICC SLE 2012, N (\%) & $10(11)$ & $2(5)$ & $1(2)$ & 0 & $1(13)$ \\
\hline EULAR-ACR 2019 criteria & $3(3)$ & 0 & $1(2)$ & $1(4)$ & 0 \\
\hline Constitutional, N (\%) & $71(80)$ & $13(30)$ & $27(44)$ & $2(8)$ & $2(25)$ \\
\hline Neuropsychiatric, N (\%) & $13(15)$ & $1(2)$ & $2(3)$ & 0 & 0 \\
\hline Mucocutaneous, N (\%) & $52(58)$ & $14(33)$ & $22(35)$ & $3(12)$ & $3(38)$ \\
\hline Serosal, N (\%) & $30(34)$ & 0 & 0 & $1(4)$ & 0 \\
\hline Musculoskeletal, N (\%) & $44(49)$ & $12(28)$ & $10(16)$ & $3(12)$ & 0 \\
\hline Renal, N (\%) & $43(48)$ & $7(16)$ & $17(27)$ & $4(16)$ & 0 \\
\hline Haematological, N (\%) & $48(54)$ & $10(23)$ & $10(16)$ & $3(12)$ & $1(13)$ \\
\hline Antiphospholipid antibodies, N (\%) & $19(21)$ & $2(5)$ & $4(6)$ & $1(4)$ & 0 \\
\hline Low complement, N (\%) & $50(56)$ & $8(19)$ & $12(19)$ & 0 & $1(13)$ \\
\hline Anti-Sm, N (\%) & &
\end{tabular}

Figure 1. Baseline characteristics and classification criteria compared across five SARD diagnoses.

The sensitivity and specificity of the EULAR19 is similar to ACR97 (sensitivity $84 \%(95 \% \mathrm{Cl} 75-91 \%)$ vs. $87 \%$ (95\% Cl $78-93 \%)$ and specificity $78 \%(95 \% \mathrm{Cl}$ $70-84 \%)$ vs. $76 \%$ (95\% Cl 68-83\%) respectively). The SLICC12 criteria by contrast are more sensitive $(94 \%(95 \% \mathrm{Cl} 87-98 \%))$ and less specific $(61 \%(95 \% \mathrm{Cl}$ $52-69 \%)$ ) in this cohort.

Figure 2 illustrates patients with a clinician diagnosis of SLE or UCTD who meet each of the classification criteria. Of the 89 patients with a clinician diagnosis of SLE, $39(44 \%)$ patients would have sufficient points to meet EULAR19 criteria on blood test results alone in the absence of clinical symptoms. Four pSS patients and 4 UCTD patients would also meet EULAR19 criteria from positive blood results alone.

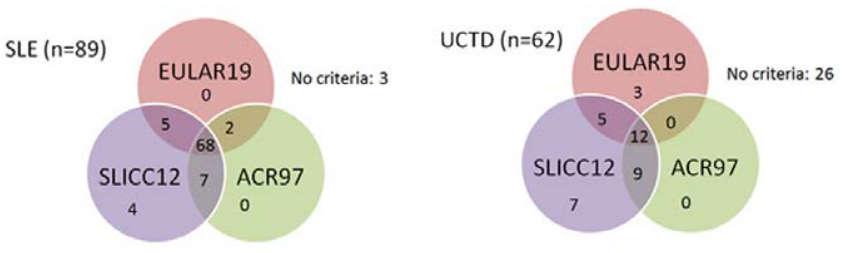

Figure 2. Venn diagrams illustrating patients with SLE and UCTD who meet the EULAR19, ACR97 and SLICC12 classification criteria. No criteria refers to the patients not meeting any of the three SLE classification criteria.

Conclusion: These results suggest that the EULAR19 criteria perform comparably to the ACR97 criteria when applied to an established cohort of SARDs.
Similar to SLICC12, it is possible to classify patients as having SLE using the EULAR19 criteria by haematological and other laboratory tests. To what extent haematological abnormalities can be potentially used as the sole 'clinical criteria' needs consideration.

\begin{tabular}{|c|c|c|c|c|c|}
\hline & SLE $\mathrm{n}=89$ & pSS $n=43$ & UCTD $n=62$ & $S S c n=25$ & Myositis $n=8$ \\
\hline Female N (\%) & $82(92)$ & $42(98)$ & $53(85)$ & $24(96)$ & $8(100)$ \\
\hline Age, mean (SD) /years & 44 (13) & $52(12)$ & $47(13)$ & $60(9)$ & $53(7)$ \\
\hline $\begin{array}{l}\text { Disease duration, mean } \\
\text { (SD) /years }\end{array}$ & $12(10)$ & $6(5)$ & $5(7)$ & $9(11)$ & $3(3)$ \\
\hline \multicolumn{6}{|l|}{ SLE classification criteria } \\
\hline $\begin{array}{l}\text { EULAR SLE 2019, } \\
\text { N (\%) }\end{array}$ & $75(84)$ & $10(23)$ & $20(32)$ & $1(4)$ & 0 \\
\hline ACR SLE 1997, N (\%) & $77(87)$ & $9(21)$ & $21(34)$ & $2(8)$ & $1(13)$ \\
\hline SLICC SLE 2012, N (\%) & $84(94)$ & $15(35)$ & $33(53)$ & $4(16)$ & $2(25)$ \\
\hline \multicolumn{6}{|l|}{ EULAR-ACR 2019 criteria } \\
\hline Constitutional, N (\%) & $10(11)$ & $2(5)$ & $1(2)$ & 0 & $1(13)$ \\
\hline Neuropsychiatric, N (\%) & 3 (3) & 0 & $1(2)$ & $1(4)$ & 0 \\
\hline Mucocutaneous, $\mathrm{N}(\%)$ & $71(80)$ & $13(30)$ & $27(44)$ & $2(8)$ & $2(25)$ \\
\hline Serosal, N (\%) & $13(15)$ & $1(2)$ & $2(3)$ & 0 & 0 \\
\hline Musculoskeletal, N (\%) & $52(58)$ & $14(33)$ & $22(35)$ & $3(12)$ & $3(38)$ \\
\hline Renal, N (\%) & 30 (34) & 0 & 0 & $1(4)$ & 0 \\
\hline Haematological, N (\%) & 44 (49) & $12(28)$ & $10(16)$ & $3(12)$ & 0 \\
\hline $\begin{array}{l}\text { Antiphospholipid anti- } \\
\text { bodies, N (\%) }\end{array}$ & $43(48)$ & $7(16)$ & 17 (27) & $4(16)$ & 0 \\
\hline Low complement, $\mathrm{N}(\%)$ & $48(54)$ & $10(23)$ & $10(16)$ & $3(12)$ & $1(13)$ \\
\hline Anti-Sm, N (\%) & $19(21)$ & $2(5)$ & $4(6)$ & $1(4)$ & 0 \\
\hline Anti-dsDNA, N (\%) & $50(56)$ & $8(19)$ & $12(19)$ & 0 & $1(13)$ \\
\hline
\end{tabular}

Disclosure of Interests: Sarah Dyball: None declared, John Reynolds: None declared, Eoghan McCarthy: None declared, Sahena Haque: None declared, Ariane Herrick: None declared, Hector Chinoy: None declared, Ellen Bruce: None declared, Ben Parker Grant/research support from: GSK and Sanofi Genzyme, Consultant of: GSK, AstraZenaca, UCV, Abbvie, Pfizer, BMS, Celltrion, Ian N. Bruce Grant/research support from: Genzyme Sanofi, GSK, and UCB, Consultant of: Eli Lilly, AstraZeneca, UCB, Iltoo, and Merck Serono, Speakers bureau: UCB

DOI: 10.1136/annrheumdis-2020-eular.851

\begin{tabular}{|l|l|}
\hline OP0095 & A DECISION MODEL OF LABIAL GLAND BIOPSY \\
BASED ON B-MODE ULTRASONOGRAPHY WITH \\
SHEAR-WAVE ELASTOGRAPHY IN PATIENTS WITH \\
SUSPECTED SJÖGREN'S SYNDROME
\end{tabular}

Y. Mo ${ }^{1}$, S. $\mathrm{Hao}^{2}$, Q. H. LI ${ }^{1}$, J. J. Liang ${ }^{1}$, Y. Luo ${ }^{2}$, J. W. Wang ${ }^{1}$, X. Zhang ${ }^{1}, \mathrm{H}$ W. Lu', L. Dai'. ' ${ }^{1}$ Sun Yat-Sen Memorial Hospital, Sun Yat-Sen University, Department of Rheumatology, Guangzhou, China; ${ }^{2}$ Sun Yat-Sen Memorial Hospital, Sun Yat-Sen University, Department of Ultrasonography, Guangzhou, China

Background: Focal lymphocytic sialadenitis defined as focus score $(F S) \geq 1$ on labial gland (LG) biopsy plays an integral role in various classification criteria of Sjögren's syndrome (SS). However, suspected patients often hesitate to receive a biopsy; and rheumatologists hope a decision for biopsy based on a high predicted incidence of $\mathrm{FS} \geq 1$, or against biopsy based on an absolutely low predicted incidence.

Objectives: To build a decision model of LG biopsy based on B-mode ultrasonography (US) with shear-wave elastography (SWE) in patients with suspected SS.

Methods: Patients who had at least one symptom of oral dryness (based on AECG questions) or had anti-SSA positive were recruited and signed a written informed consent. Bilateral parotid (PG) and submandibular glands (SMG) were examined with B-mode US which graded the echostructure of each gland on a scoring system scaled 0 to 4 (US score), and SWE which described the elasticity of glands. Then LG biopsy was performed.

Results:

(1) Ninety-one patients whose mean age was $43 \pm 15$ years were enrolled and $93 \%$ of them were female. Anti-SSA was detected in 77 patients $(85 \%)$ and 28 patients $(31 \%)$ showed unstimulated whole saliva flow rate (USFR) $\leq 0.1 \mathrm{~mL} / \mathrm{mim}$. There were 57 patients $(63 \%)$ showing $\mathrm{FS} \geq 1$ on LG biopsy. Sixty-three patients $(69 \%)$ were classified as primary SS, 10 patients (10\%) were secondary SS, 18 patients $(20 \%)$ were UCTD and one patient was RA without SS.

(2) US scores were equal between PG and SMG in 59 patients (65\%), while the rest patients showed different US scores between two glands: 7 patients (8\%) showed higher US scores in PG and 25 patients $(27 \%)$ showed higher scores in SMG. In each pair of glands US scores were equal. SWE values in PG or SMG of US score 1, 2 or 3 were significantly 
higher than those of US score 0 , while SWE values in glands of US score 4 became declined and showed no significant difference from those with US score 0 (Figure 1A)

(3) Heatmap showed US scores in either major salivary gland of patients with $F S \geq 1$ on LG biopsy were significantly higher than those with $F S<1$ (all $p<0.001$, Figure 1B). ROC curve showed a total US score (including bilateral $P G$ and $S M G$ ) $\geq 9$ and a total $S W E$ value (including bilateral $P G$ and $S M G) \geq 30$ could significantly recognize patients with $F S \geq 1$, respectively with specificity of $100 \%$ and $93 \%$ (Figure 1C). In this cohort, among 51 patients with a total US score $\geq 9$ and/or a total SWE value $\geq 30,49$ patients (96\%) showed FS $\geq 1$ on LG biopsy; while two outliers showed total US scores were both 8 although combined SWE values $\geq 30$. Other 29 patients showed total US scores $\leq 6$ with total SWE values $<30$ and only one patient (3\%) showed $\mathrm{FS} \geq 1$ on $\mathrm{LG}$ biopsy. The remaining 11 patients showed total US scores were 8 with total SWE values $<30$ and $64 \%$ of them $(n=7)$ showed $F S \geq 1$

Conclusion: A preliminary decision model of LG biopsy based on B-mode US with SWE in patients with suspected SS were built in Table 1. For example, rheumatologists should reassess the need for biopsy if the incidence of $\mathrm{FS} \geq 1$ would be $<5 \%$. Another cohort of patients with suspected SS is needed for further validation

Table 1. A preliminary decision model of LG biopsy based on B-mode US with SWE in patients with suspected SS

\begin{tabular}{ll}
\hline Algorithm $^{*}$ & Comments on the decision of LG biopsy \\
\hline $\begin{array}{l}\text { A total US score } \geq 9 \text { and/or a } \\
\text { total SWE } \geq 30\end{array}$ & $\begin{array}{l}\text { The specificity of } F S \geq 1 \text { on biopsy is }>93 \% \text {. Biopsy is rec- } \\
\text { ommended. In some special cases (e.g. contraindicated to } \\
\text { biopsy), this item is a potential alternative to LG biopsy. } \\
\text { It is hard to predict the result of FS, so biopsy is strongly } \\
\text { recommended. }\end{array}$ \\
$\begin{array}{l}\text { A total US score 7 8 with a } \\
\text { total SWE }<30\end{array}$ & $\begin{array}{l}\text { The incidence of FS } \geq 1 \text { would be }<5 \% \text {. Rheumatologists } \\
\text { should reassess the need for biopsy. }\end{array}$ \\
total SWE $<30$ &
\end{tabular}
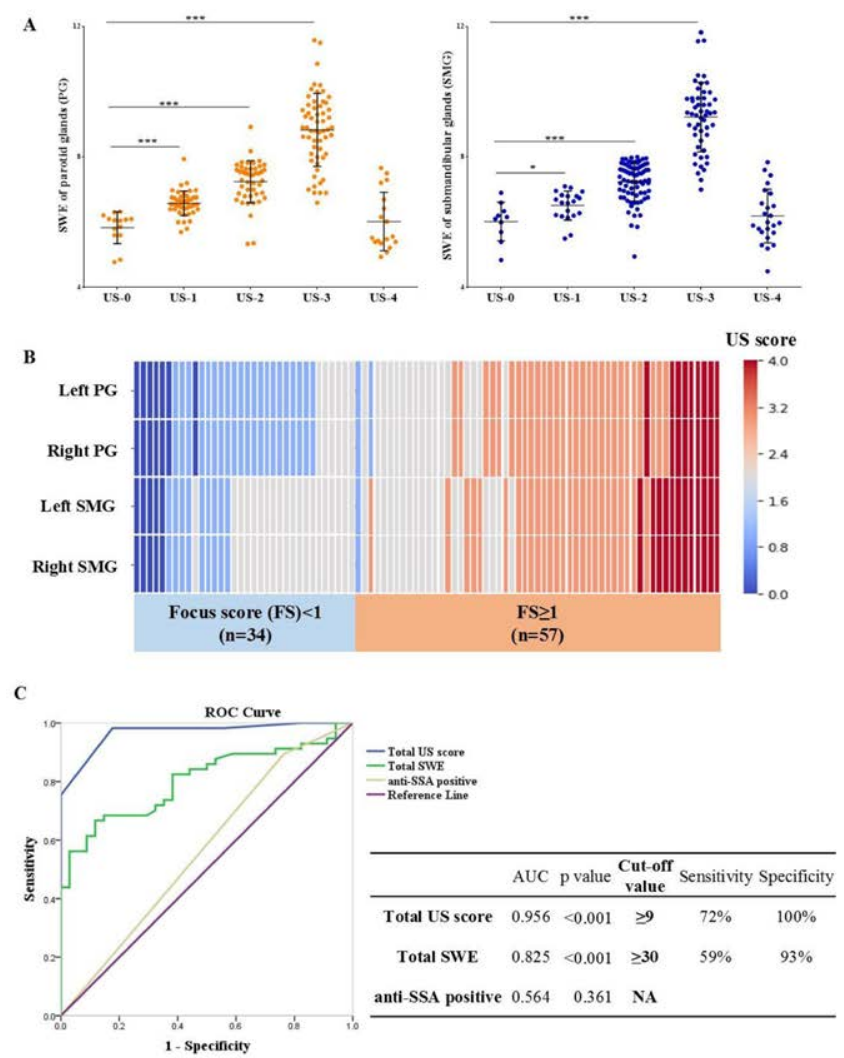

Figure 1 B-mode ultrasonography (US) with shear-wave elastography (SWE) in 91 patients with suspected SS. A: SWE values in 182 parotid glands (PG, left) or 182 submandibular glands (SMG, right) suspected $5 S$. A. SW of US score 0 to . B. Heatmap showed US scores in either major salivary gland of 57 patients with $\mathrm{FS} \geq 1$ on labial gland biopsy a 34 pants $P$ P $<1$. and anti-SSA positive on the incidence of $\mathrm{FS} \geq 1$ on labial gland biopsy.
References: None

Disclosure of Interests: None declared

DOI: 10.1136/annrheumdis-2020-eular.3493

\section{OP0096 THE DIFFERENCES BETWEEN SJÖGREN'S SYNDROME PATIENTS WITH COMBINED SERONEGATIVITY AND ANTI-RO/SSA SEROPOSITIVITY}

L. Chatzis ${ }^{1}$, V. Pezoulas ${ }^{2}$, F. Ferro ${ }^{3}$, V. Donati ${ }^{3}$, A. Venetsanopoulou', E. Zampeli ${ }^{4}$, M. Mavromati ${ }^{4}$, P. Voulgari ${ }^{5}$, C. Mavragani ${ }^{1}$, D. Fotiadis ${ }^{2}$, F. Skopouli ${ }^{4}$, S. De Vita ${ }^{6}$, C. Baldini ${ }^{3}$, H. M. Moutsopoulos ${ }^{4}$, A. Tzioufas ${ }^{1}$, A. Goules ${ }^{1} .{ }^{1}$ Pathophysiology Department, Athens School of Medicine, National and Kapodistrian University of Athens, Athens, Greece, Athens, Greece; ${ }^{2}$ Unit of Medical Technology and Intelligent Information Systems, University of loannina, Ioannina, Greece, Ioannina, Greece; ${ }^{3}$ Rheumatology Unit, Department of Clinical and Experimental Medicine, University of Pisa, Italy, Pisa, Italy; ${ }^{4}$ Department of Nutrition and Clinical Dietetics, Harokopio University of Athens, Athens, Greece, ${ }^{5}$ Rheumatology Clinic, Department of Internal Medicine, Medical School, University of loannina, Ioannina, Greece, Ioannina, Greece; ${ }^{6}$ Rheumatology Clinic, Department of Medical area, University of Udine, Udine, Italy, Udine, Italy

Background: Sjögren's syndrome (SS) is characterized by B cell hyperactivity reflected by hypergammaglobulinemia as well as a plethora of autoantibodies including antinuclear antibodies (ANA), anti-Ro/SSA, anti-La/SSB and rheumatoid factors (RF). Previous studies have focused on the phenotype of single positive (ANA or anti-Ro/SSA or anti-La/SSB) or double positive (anti-Ro/SSA and anti-La/SSB positive) SS patients, showing differences regarding the age of diagnosis, sicca manifestations and specific extraglandular manifestations. To our knowledge, no study has ever explored the clinical spectrum of triple seronegative (anti-Ro/SSA + anti-La/SSB + RF negative) and quadruple seronegative (ANA +anti-Ro/SSA + anti-La/SSB + RF negative) SS patients.

Objectives: To study the differences in the clinical phenotype of triple and quadruple seronegative (SS) patients in a large cohort of well characterized patients, after comparison with anti-Ro/SSA positive patients.

Methods: From a total cohort of 1723 consecutive SS patients who fulfill the 2016 EULAR/ACR criteria and are followed up in 4 clinical centers ([Universities of Pisa and Athens, Harokopio and Ioannina, (PAHI)], those who have been found triple or quadruple seronegative were identified and compared with matched anti-Ro/SSA positive SS patients according to age of SS onset, disease duration and gender, in 1:1 and 1:2 ratio respectively. Glandular (dry mouth, dry eyes, parotid gland enlargement) and extra-glandular manifestations (Raynaud's phenomenon, chronic fatigue arthralgias/myalgias, arthritis, palpable purpura, liver involvement, kidney involvement, lung involvement, neurologic involvement, long standing lymphadenopathy and lymphoma) were compared between the 2 seronegative groups and the anti-Ro/SSA positive control group. Statistical analysis for categorical variables was performed by Fisher exact or chi-square tests and for continuous variables with $t$ test or Mann-Whitney accordingly.

Results: Two hundred and four SS patients $(11,8 \%)$ were identified as triple negatives and $53(3,0 \%)$ as quadruple, with a median disease duration of 6 years (range: 0-41) and 5 years (range: 0-32) respectively. The matched anti-Ro/SSA controls were 204 for the triple and 103 for the quadruple negatives. Triple negatives had lower frequency of monoclonal gammopathy $(5,5 \%$ vs $12,1 \% p=0,04)$, low $\mathrm{C} 4$ serum levels $(23 \%$ vs $36 \%, p=0,009)$ and lymphoma $(3,4 \%$ vs $9,8 \%$, OR= $3,06,95 \% \mathrm{Cl}=1,27-7,85)$ while quadruple negatives exhibited higher prevalence of dry eyes (100\% vs $90 \%)$ and lower prevalence of long standing lymphadenopathy $(2,7 \%$ vs $19,5 \%, p=0,001)$ and lymphoma $(0 \%$ vs $15 \%, p=0,001)$ compared to anti-Ro/SSA matched controls.

Conclusion: Combined seronegativity account for more than $10 \%$ of SS population and is associated with lower prevalence of lymphoma compared to anti-Ro/ SSA positive patients.

Disclosure of Interests: Loukas Chatzis: None declared, Vasileios Pezoulas: None declared, Francesco Ferro: None declared, Valentina Donati: None declared, Aliki Venetsanopoulou: None declared, Evangelia Zampeli: None declared, Maria Mavromati: None declared, Paraskevi Voulgari: None declared, Clio Mavragani: None declared, Dimitris Fotiadis: None declared, Fotini Skopouli: None declared, Salvatore De Vita Consultant of: Roche, Human Genome Science, Glaxo Smith Kline and Novartis, Chiara Baldini: None declared, Haralampos M. Moutsopoulos: None declared, Athanasios Tzioufas: None declared, Andreas Goules: None declared DOI: 10.1136/annrheumdis-2020-eular.4203 